PROCEEDINGS OF THE

AMERICAN MATHEMATICAL SOCIETY

Volume 135, Number 11, November 2007, Pages 3525-3535

S 0002-9939(07)08877-6

Article electronically published on July 3, 2007

\title{
A NEW $L^{\infty}$ ESTIMATE IN OPTIMAL MASS TRANSPORT
}

\author{
G. BOUCHITTÉ, C. JIMENEZ, AND M. RAJESH
}

(Communicated by David Preiss)

\begin{abstract}
Let $\Omega$ be a bounded Lipschitz regular open subset of $\mathbb{R}^{d}$ and let $\mu, \nu$ be two probablity measures on $\bar{\Omega}$. It is well known that if $\mu=f d x$ is absolutely continuous, then there exists, for every $p>1$, a unique transport map $T_{p}$ pushing forward $\mu$ on $\nu$ and which realizes the Monge-Kantorovich distance $W_{p}(\mu, \nu)$. In this paper, we establish an $L^{\infty}$ bound for the displacement map $T_{p} x-x$ which depends only on $p$, on the shape of $\Omega$ and on the essential infimum of the density $f$.
\end{abstract}

\section{Introduction AND MAIN RESUlts}

The theory of mass transportation goes back to the original works by Monge in 1781 [11] and later by Kantorovich [10. Recently a renewed interest for this theory arose in different areas of applied mathematics like economic sciences, fluid mechanics, shape optimization, signal theory, image and data compression, as well as in geometric functional analysis and large deviation theory.

In this paper, we will consider the most usual case for which the transport cost function is convex of the form $c(x, y)=|x-y|^{p}$ where $p \geq 1$ and $|\cdot|$ denotes the Euclidian norm in $\mathbb{R}^{d}$. Given two probability measures $\mu, \nu$ on $\mathbb{R}^{d}$, the associated Monge -Kantorovich mass transport problem can be written as follows:

$$
\mathcal{T}_{p}(\mu, \nu):=\inf \left\{\int_{\mathbb{R}^{d} \times \mathbb{R}^{d}}|x-y|^{p} \gamma(d x d y): \gamma \in \Gamma(\mu, \nu)\right\},
$$

where the infimum is taken over the class $\Gamma(\mu, \nu)$ of probability measures on $\mathbb{R}^{d} \times \mathbb{R}^{d}$ whose marginals are respectively $\mu$ and $\nu$. It turns out that the infimum above is finite and achieved, provided $\mu, \nu$ have finite $p^{\text {th }}$-order moments. The $p$-Wasserstein distance between such elements $\mu, \nu$ is then defined by

$$
W_{p}(\mu, \nu):=\left(\mathcal{T}_{p}(\mu, \nu)\right)^{\frac{1}{p}},
$$

and the minimizing measure $\gamma$ is called optimal planning.

It is well known that if one of the measures, say $\mu$, is absolutely continuous with respect to the Lebesgue measure, then the following equality holds:

$$
\mathcal{T}_{p}(\mu, \nu)=\inf \left\{\int|x-T x|^{p} \mu(d x): T^{\sharp}(\mu)=\nu\right\},
$$

Received by the editors January 9, 2006 and, in revised form, June 23, 2006.

2000 Mathematics Subject Classification. Primary 39B62, 46N10, 49Q20.

Key words and phrases. Wasserstein distance, optimal transport map, uniform estimates.

(C)2007 American Mathematical Society Reverts to public domain 28 years from publication 
where the infimum is searched among all transports maps $T: X \mapsto Y$ pushing forward $\mu$ on $\nu$ (i.e. such that $\mu\left(T^{-1}(B)\right)=\nu(B)$ for all Borel subsets $B \subset Y$ ). In fact this new problem is equivalent to restricting the infimum in (1.1) to the subclass $\left\{\gamma_{T}\right\} \subset \Gamma(\mu, \nu)$ where $\left\langle\gamma_{T}, \phi(x, y)\right\rangle:=\int_{X} \phi(x, T x) \mu(d x)$. The formulation (1.2) is the original setting of the transport problem proposed by Monge in the case $p=1$, for which the existence of an optimal transport map $T$ is a difficult question solved recently (see [4, 6], 8, [12 ). Assuming that $\mu$ is an absolutely continuous measure, it is now well known that such an optimal map exists whenever $p \geq 1$. Futhermore it is unique if $p>1$, and in this case we will denote it by $T_{p}(\mu, \nu)$. Notice that the associated transport plan $\gamma_{T_{p}}$ will be the unique solution for (1.1) as well (see for instance [9]).

In this paper, we address the problem of stability of the optimal transport map $T_{p}(\mu, \nu)$. Let $\mu$ be given as before. If $\left\{\nu^{(h)}\right\}$ is a sequence of probability measures on $\mathbb{R}^{d}$ such that $W_{p}\left(\mu, \nu^{(h)}\right) \rightarrow 0$ (this implies that $\nu_{h} \rightarrow \mu$ tightly), then it is easy to see that the optimal $T^{(h)}:=T_{p}\left(\mu, \nu_{h}\right)$ does converge in measure (with respect to $\mu$ ) to the identity map, i.e.

$$
\forall \varepsilon>0 \quad, \quad \mu\left(\left\{x:\left|T^{(h)}(x)-x\right|>\varepsilon\right\}\right) \rightarrow 0 \quad \text { as } h \rightarrow \infty .
$$

We will prove that in fact the convergence of $T^{(h)}$ to the identity is uniform on any compact regular subset of $\mathbb{R}^{d}$ where the density of $\mu$ has a positive lowerbound. More precisely, we will establish the following estimate:

Proposition 1.1. Let $p>1$ and $\mu=f d x$ be an absolutely continuous probability measure on $\mathbb{R}^{d}$ such that $\int|x|^{p} \mu(d x)<+\infty$. Let $a>0$ and let $\Omega$ be a bounded convex open subset of $\mathbb{R}^{d}$ such that $f \geq a$ a.e. on $\Omega$. Then, for every Borel probability measure $\nu$ on $\bar{\Omega}$, the optimal transport map $T_{\nu}:=T_{p}(\mu, \nu)$ satisfies the inequality

$$
\left\|I d-T_{\nu}\right\|_{L^{\infty}(\Omega)}^{p+d} \leq c_{p, d}(\Omega) \frac{W_{p}^{p}(\mu, \nu)}{a}
$$

where $c_{p, d}(\Omega)$ is a positive constant depending only on $p, d$ and $\Omega$.

The result of Proposition 1.1 can be stated in a more intrinsic (but weaker) form by minorizing the left hand side of (1.3) in terms of the $W_{\infty}$ distance of $\mu$ to $\nu$. This distance is defined as follows:

$$
W_{\infty}(\mu, \nu):=\inf \{t>0: \exists \gamma \in \Gamma(\mu, \nu),|x-y| \leq t \quad \gamma \text { a.e. }\} .
$$

The main result of this paper is the following:

Theorem 1.2. Let $\Omega$ be a bounded connected open subset of $\mathbb{R}^{d}$ with $C^{0,1}$ boundary and denote by $\mathcal{P}(\bar{\Omega})$ (resp $\mathcal{P}_{\mathrm{ac}}(\Omega)$ ) the set of Borel (resp. absolutely continuous) probability measures on $\bar{\Omega}$. Then, for every $p>1$, and every pair $(\mu, \nu) \in \mathcal{P}_{\text {ac }}(\Omega) \times$ $\mathcal{P}(\bar{\Omega})$ there holds

$$
\left(W_{\infty}(\mu, \nu)\right)^{p+d} \leq C_{p, d}(\Omega)\left\|f^{-1}\right\|_{L^{\infty}(\Omega)} W_{p}^{p}(\mu, \nu)
$$

where $f=\frac{d \mu}{d x}$ and $C_{p, d}(\Omega)$ is a positive constant depending only on $p, d$ and $\Omega$. 
Remark 1.3. We remark that in Theorem 1.2, the convexity assumption of Proposition 1.1 has been dropped. We believe that the constant $C_{p, d}(\Omega)$ found in the proof is not sharp. The optimal constant can be seen as the following shape function:

$$
\tilde{C}_{p, d}(\Omega):=\sup \left\{\frac{\left(W_{\infty}(\mu, \nu)\right)^{p+d}}{W_{p}^{p}(\mu, \nu)\left\|\left(\frac{d \mu}{d x}\right)^{-1}\right\|_{L^{\infty}(\Omega)}}:(\mu, \nu) \in \mathcal{P}_{\mathrm{ac}}(\Omega) \times \mathcal{P}(\bar{\Omega})\right\} .
$$

Notice that this function is translation and dilation invariant. To check that, for $\lambda>0$, we have $\tilde{C}_{p, d}(\lambda \Omega)=\tilde{C}_{p, d}(\Omega)$, it is enough to consider the push forward of $\mu, \nu$ through the map $S_{\lambda}: x \rightarrow \lambda x$ and to notice that

$$
W_{p}\left(S_{\lambda}^{\sharp} \mu, S_{\lambda}^{\sharp} \nu\right)=\lambda W_{p}(\mu, \nu), p \in[1,+\infty] \quad, \quad S_{\lambda}^{\sharp} \mu=\frac{1}{\lambda^{d}} f\left(\frac{x}{\lambda}\right) d x .
$$

Remark 1.4. By Hölder inequality, $W_{p}(\mu, \nu)$ as a function of $p$ is monotone nondecreasing. Recalling the definition (1.4), it is then easy to check that

$$
W_{\infty}(\mu, \nu)=\lim _{p \rightarrow \infty} W_{p}(\mu, \nu)=\sup _{p \geq 1} W_{p}(\mu, \nu) .
$$

Then the estimate (1.5) implies that

$$
C_{p, d}(\Omega) \geq \frac{W_{\infty}^{d}(\mu, \nu)}{\left\|f^{-1}\right\|_{L^{\infty}(\Omega)}} \quad \forall(\mu, \nu) \in \mathcal{P}_{\mathrm{ac}}(\Omega) \times \mathcal{P}(\bar{\Omega}) .
$$

The supremum of the right hand side of the previous inequality is obtained by taking $f$ to be constant and $\nu$ a Dirac mass. If $D$ denotes the diameter and $|\Omega|$ the Lebesgue measure of $\Omega$, we derive that

$$
C_{p, d}(\Omega) \geq \frac{D^{d}}{|\Omega|}
$$

showing that $C_{p, d}(\Omega)$ blows up when $\Omega$ becomes thinner and thinner.

Let us finally notice that the inequality $W_{p}^{p}(\mu, \nu) \geq D^{p-q} W_{q}^{q}(\mu, \nu)$ valid for $q \geq p$ (due to $|x-y|^{p} \leq D^{q-p}|x-y|^{q}$ for all $(x, y) \in \Omega^{2}$ ) is not useful for our purpose since, passing to the limit as $q \rightarrow \infty$, we merely obtain the trivial inequality $W_{\infty}(\mu, \nu) \leq D$.

Remark 1.5. It turns out that the constant $c_{p, d}(\Omega)$ we find in the proof of Proposition 1.1 (see (2.19) and Remark 2.6 below) blows up as $p \rightarrow 1$. It is well known that in the case $p=1$, the optimal transport map still exists but is not unique. In fact it is easy to check (see Example 2.7) that the inequality (1.5) cannot be valid for $p=1$ if we choose particular optimal transport maps. However, the inequality still holds in the one dimensional case if we select the unique monotone tranport $T_{1}(\mu, \nu)$. We strongly believe that the same conclusion holds true in higher dimension if we use the extended concept of the monotone transport map introduced by V.N. Sudakov and later by L. Ambrosio (see [12] and [4) (which agrees with the limit of $T_{p}(\mu, \nu)$ as $\left.p \rightarrow 1\right)$. Accordingly, we conjecture that Theorem 1.2 holds true for $p=1$ which amounts to saying that the shape function defined in (1.6) does not blow up as $p \rightarrow 1$.

Remark 1.6. Many regularity results for the optimal transport map are known in the case where $p=2$ and $\mu, \nu$ both belong to $\mathcal{P}_{\text {ac }}(\Omega)$. Indeed in this case the optimal $T$ is the gradient of a convex potential $\varphi$ solving the Monge Ampere equation $\operatorname{det} D^{2} \varphi=\frac{f}{g(\nabla \varphi)}$ on $\Omega$ where $f, g$ are the respective densities of $\mu$ and $\nu$. Then by Caffarelli's regularity result ([1, 2]), which holds under lower bounds assumptions 
on $f, g$ and a convexity assumption on their support $\Omega$, the potential $\varphi$ inherits the regularity of $f$ and $g$ : if $f, g$ are $C^{0, \alpha}$ Hölder continuous, then $\varphi$ is $C^{2, \alpha}$ and $T=\nabla \varphi \in C^{1, \alpha}$. We expect that such kinds of results could lead to similar estimates as in Proposition 1.1 when the data $\mu$ and $\nu$ are smooth densities. However nothing essential seems to be known if $p \neq 2$ or if $\nu$ is a singular measure. In particular in optimal location problems (see for instance [5]), purely atomic measures of the kind $\nu=\sum c_{i} \delta_{x_{i}}$ arise naturally and the associated optimal transport map $T$ induces a partition of $\Omega$ by the sets $A_{i}=T^{-1}\left(\left\{x_{i}\right\}\right)$. Our $L^{\infty}$ estimate in Proposition 1.1 provides a very useful upper bound for the diameter of the $A_{i}$ 's.

\section{Proofs And examples}

The basic argument to prove Proposition 1.1 will be the $p$-monotonicity property of the optimal transport. First we shall prove some preliminary results in the form of lemmas. The scalar product and the Euclidean norm in $\mathbb{R}^{d}$ are denoted repectively by the symbols $(\cdot \mid \cdot)$ and $|\cdot|$.

Lemma 2.1. Let $p>1, \mu, \nu$ be as in Proposition 1.1 and let $T:=T_{p}(\mu, \nu)$ be the optimal map related to $W_{p}(\mu, \nu)$. Then, there exists a $\mu$-negligible subset $N \subset \mathbb{R}^{d}$ such that, for every $(x, y) \in\left(\mathbb{R}^{d} \backslash N\right)^{2}$, there holds the inequality

$$
|x-T(x)|^{p}+|y-T(y)|^{p} \leq|x-T(y)|^{p}+|y-T(y)|^{p} .
$$

Proof. It is a consequence of the $p$-cyclic monotonicity of the support of the optimal transport plan $\gamma_{T_{p}(\mu, \nu)}$ [3, 9] and of the fact that any $\gamma$-negligible set has a $\mu$ negligible first projection.

In view of (2.1), it looks natural to introduce the function

$$
\psi_{p}(x, y, z):=\inf _{w \in \mathbb{R}^{d}}\left\{|w-z|^{p}:|w-z|^{p}+|x-y|^{p} \leq|z-y|^{p}+|x-w|^{p}\right\} .
$$

It is easy to check that $\psi_{p}$ enjoys the following properties for every $x, y, z \in \mathbb{R}^{d}$ :

(i) $\quad \psi_{p}(x, y, z)=\psi_{p}(x+a, y+a, z+a), \quad$ for every $a \in \mathbb{R}^{d}$,

(ii) $\quad \psi_{p}(x, y, z)=\psi_{p}(Q x, Q y, Q z), \quad$ for every isometry $Q \in \mathrm{SO}(d)$,

(iii) $\psi_{p}(\lambda x, \lambda y, \lambda z)=|\lambda|^{p} \psi_{p}(x, y, z), \quad$ for every $\lambda \in \mathbb{R}$

(iv) $\quad \psi_{p}(x, x, z)=0$.

Thanks to (i) and (iii), for all $x, y \in \mathbb{R}^{d}$ with $x \neq y$, we derive that

$$
\psi_{p}(x, y, z)=|y-x|^{p} \bar{\psi}_{p}\left(\frac{y-x}{|y-x|}, \frac{z-x}{|y-x|}\right) \quad \text { where } \quad \bar{\psi}_{p}(u, z):=\psi_{p}(0, u, z) \text {. }
$$

This normalized function $\bar{\psi}_{p}(u, z)$ defined on $S^{d-1} \times \mathbb{R}^{d}$ can be rewriten as

$$
\overline{\psi_{p}}(u, z)=\inf \left\{|w-z|^{p}: w \in \Gamma_{p}(u, z)\right\}
$$

where

$$
\Gamma_{p}(u, z):=\left\{w \in \mathbb{R}^{d}:|w-z|^{p}+1 \leq|z-u|^{p}+|w|^{p}\right\} .
$$

Lemma 2.2. The function $\overline{\psi_{p}}$ is lower semi-continuous on $S^{d-1} \times \mathbb{R}^{d}$. It is strictly positive if and only if $z$ belongs to the open p-ellipsoid

$$
Q_{p}(u):=\left\{z \in \mathbb{R}^{d}:|z|^{p}+|z-u|^{p}<1\right\} .
$$


One can readily check that the $p$-ellipsoid $Q_{p}(u)$ defined above shrinks as $p$ goes down to 1 and that $Q_{p}(u)$ is nonempty if and only if $p>1$. It turns out that the function $\overline{\psi_{p}}$ is not continuous. For instance, in the case $p=2, Q_{2}(u)$ is the ball of radius $1 / 2$ centered at $u / 2$ and the following explicit expression holds:

$$
\overline{\psi_{2}}(u, z)= \begin{cases}\left(|z|-\frac{(z \mid u)}{|z|}\right)^{2} & \text { if } 0<(z \mid u)<|z|^{2} \\ 0 & \text { otherwise. }\end{cases}
$$

Proof. Clearly the minimum in (2.5) is achieved as the distance of $z$ to the closed subset $\Gamma_{p}(u, z)$ (which is nonempty since it contains $\{u\}$ ). It follows that a vanishing minimum forces $z \notin Q_{p}(u)$. It remains to check the lower semicontinuity: let $\left(u_{n}, z_{n}\right) \rightarrow(u, z)$ and assume without loss of generality that $\overline{\psi_{p}}\left(u_{n}, z_{n}\right) \rightarrow \alpha<+\infty$. Choose $w_{n} \in \Gamma\left(u_{n}, z_{n}\right)$ so that $\overline{\psi_{p}}\left(u_{n}, z_{n}\right)=\left|w_{n}-z_{n}\right|^{p}$. Then, since $0 \in \Gamma_{p}\left(u_{n}, z_{n}\right)$, we have $\left|w_{n}-z_{n}\right| \leq\left|z_{n}\right|^{p}$ yielding that $\left\{w_{n}\right\}$ is bounded. Let $w$ be a cluster point; then obviously $w \in \Gamma_{p}(u, z)$ and $\alpha=|w-z|^{p} \geq \overline{\psi_{p}}(u, z)$.

Eventually, let us consider, for every unit vector $u \in S^{d-1}$ and every $L>0$, the convex cone

$$
\Lambda_{L}(u):=\left\{y \in \mathbb{R}^{d}:(y \mid u) \geq L \sqrt{|y|^{2}-(y \mid u)^{2}}\right\} .
$$

Lemma 2.3. Let $\Omega$ be a bounded open subset in $\mathbb{R}^{d}$ with $C^{0,1}$ boundary. Then we can find a constant $L>0$ such that, for every $x \in \partial \Omega$, there exists $u_{x} \in S^{d-1}$ satisfying the following property:

$$
\liminf _{\delta} \theta_{\delta} \geq \theta_{L, x} \quad \text { whenever } x_{\delta} \in \Omega \text { and } x_{\delta} \rightarrow x \text { as } \delta \rightarrow 0,
$$

$\theta_{\delta}$ and $\theta_{L, x}$ being the characteristic functions of the sets $\frac{\Omega-x_{\delta}}{\delta}$ and $\Lambda_{L}\left(u_{x}\right)$.

Notice that, if $x \in \Omega$, we obviously obtain:

$$
x_{\delta} \in \Omega, \quad x_{\delta} \rightarrow x \Longrightarrow \liminf _{\delta} \theta_{\delta}(z) \geq 1, \forall z \in \mathbb{R}^{d} .
$$

Proof. Let $x \in \partial \Omega$ and let $x_{\delta} \in \Omega$ such that $x_{\delta} \rightarrow x$. As the boundary of $\Omega$ is compact and Lipschitz, there exists a constant $L>0$ (independent of $x$ ), a pair $\left(u_{x}, r_{x}\right) \in S^{d-1} \times(0,+\infty)$ and an $L$-Lipschitz map $\gamma_{x}: \mathbb{R}^{d-1} \rightarrow \mathbb{R}$ such that, upon a translation and a rotation of the coordinate axes, we have $u_{x}=(0, \ldots, 1), \gamma_{x}(0)=0$ and

$$
\Omega \cap B\left(x, r_{x}\right)=\left\{y \in \mathbb{R}^{d}:|y|<r_{x}, \gamma_{x}\left(y_{1}, \ldots, y_{d-1}\right)<y_{d}\right\}
$$

(here $B\left(x, r_{x}\right)$ denotes the ball centered at $x$ of radius $r_{x}$ ).

Assuming that $z \in \Lambda_{L}\left(u_{x}\right)$, we are reduced to prove that $x_{\delta}+\delta z$ belongs to $\Omega$ for $\delta$ sufficiently small. In the new coordinates, we write $z$ as $\left(z^{\prime}, z_{d}\right)\left(z^{\prime} \in \mathbb{R}^{d-1}\right)$ with $z_{d} \geq L\left|z^{\prime}\right|$. As $x_{\delta} \in \Omega$, by (2.9), we have $\left(x_{\delta}\right)_{d}>\gamma_{x}\left(x_{\delta}^{\prime}\right)$ and since $\gamma_{x}$ is $L$-Lipschitz,

$$
\gamma_{x}\left(x_{\delta}^{\prime}+\delta z^{\prime}\right) \leq \gamma_{x}\left(x_{\delta}^{\prime}\right)+L \delta\left|z^{\prime}\right|<\left(x_{\delta}\right)_{d}+z_{d} .
$$

The conclusion follows from (2.9) by noticing that $x_{\delta} \in B\left(x, r_{x}\right)$ for small $\delta$. 
We introduce the function

$$
\rho(x, y):=\frac{\int_{\Omega} \psi_{p}(x, y, z) d z}{|x-y|^{p+d}}
$$

and, for every $t>0$, we set:

$$
\omega(t):=\inf \{\rho(x, y):(x, y) \in \bar{\Omega} \times \bar{\Omega},|x-y|=t\} .
$$

By Lemma 2.2 and (2.4) $\rho$ is lower semi-continuous on $\bar{\Omega} \times \bar{\Omega} \backslash \Delta$ where $\Delta:=$ $\{(x, x): x \in \bar{\Omega}\}$. By the compactness of $\bar{\Omega}$, it follows that the function $\omega(t)$ is lower semicontinuous on the interval $(0, D]$ where $D$ denotes the diameter of $\Omega$.

A consequence of Lemma 2.3 is the following:

Lemma 2.4. Let $\Omega$ be a bounded open subset in $\mathbb{R}^{d}$ with $C^{0,1}$ boundary. Then:

$$
\omega_{p, d}(\Omega):=\liminf _{t \rightarrow 0^{+}} \omega(t)>0 .
$$

Proof. Let us proceed by contradiction assuming that $\omega_{p, d}(\Omega)=0$. Then there exists $\left\{\left(x_{n}, y_{n}\right)\right\}$ a sequence in $\bar{\Omega}^{2} \backslash \Delta$ such that $\left|x_{n}-y_{n}\right|:=\frac{1}{n} \rightarrow 0$ and $\rho\left(x_{n}, y_{n}\right) \rightarrow$ 0 . Possibly passing to a subsequence we may assume that $x_{n}$ and $y_{n}$ both converge to the same limit $x \in \bar{\Omega}$ and that $u_{n}:=\frac{y_{n}-x_{n}}{\left|y_{n}-x_{n}\right|}$ converges to an element $u_{0} \in$ $S^{d-1}$. Then exploiting (2.4) and recalling (2.10), we can rewrite after the change of variables $w=n(z-x)$ :

$$
\rho\left(x_{n}, y_{n}\right)=\int_{n\left(\Omega-x_{n}\right)} \overline{\psi_{n}}\left(u_{n}, w\right) d w .
$$

Assume first that $x \in \partial \Omega$ and let $u_{x}$ be the associated unit vector given in Lemma 2.3 . Then by the lower semicontinuity of $\overline{\psi_{p}}$ and by (2.7), we deduce from Fatou's lemma that

$$
0=\liminf _{\delta \rightarrow 0} \rho\left(x_{n}, y_{n}\right) \geq \int_{\Lambda_{L}\left(u_{x}\right)} \overline{\psi_{p}}\left(u_{0}, w\right) d w .
$$

In fact, we do not know if the direction of the unit vector $u_{0}$ is correlated with that of the outwards normal $u_{x}$ and the right hand side integral in (2.12) might vanish. To prevent this, it is enough to consider the alternative change of variables $w=n\left(z-y_{n}\right)$ in (2.10) so that, exploiting properties i) and iii) in (2.3), we have

$$
\rho\left(x_{n}, y_{n}\right)=\int_{n\left(\Omega-y_{n}\right)} \overline{\psi_{p}}\left(u_{n}, u_{n}+w\right) d w .
$$

Then passing to the limit in $\delta$, we also obtain

$$
0=\int_{\Lambda_{L}\left(u_{x}\right)} \overline{\psi_{p}}\left(u_{0}, u_{0}+w\right) d w .
$$

By Lemma 2.2 the function $\overline{\psi_{p}}\left(u_{0}, \cdot\right)$ is strictly positive on $Q_{p}\left(u_{0}\right)$ and the function $\overline{\psi_{p}}\left(u_{0}, u_{0}+\cdot\right)$ is strictly positive on $Q_{p}\left(u_{0}\right)-u_{0}=-Q_{p}\left(u_{0}\right)$. It can be seen that any cone $\Lambda_{L}\left(u_{x}\right)$ intersects at least one of the domains $Q_{p}\left(u_{0}\right)$ and $-Q_{p}\left(u_{0}\right)$ in a set of positive measure. This contradicts either (2.12) or (2.13).

Eventually, the same contradiction occurs if $x$ belongs to $\Omega$ since, by (2.8), we would obtain that $\int_{\mathbb{R}^{d}} \overline{\psi_{p}}\left(u_{0}, w\right) d w=\int_{\mathbb{R}^{d}} \overline{\psi_{p}}\left(u_{0}, u_{0}+w\right) d w=0$. The proof of the Lemma 2.4 is achieved. 
Lemma 2.5. Let $\Omega$ be a bounded connected open subset in $\mathbb{R}^{d}$ with $C^{0,1}$ boundary. Let $\rho_{0}, \rho_{1}$ be two elements of $\mathcal{P}(\bar{\Omega})$. Then there exist a parametrized curve $t \in$ $[0,1] \mapsto \rho_{t} \in \mathcal{P}(\bar{\Omega})$ connecting $\rho_{0}$ to $\rho_{1}$ and a constant $k=k(\Omega)>0$ (depending only on $\Omega$ ) such that, for all $s, t \in[0,1]$, there holds:

$$
W_{\infty}\left(\rho_{s}, \rho_{t}\right) \leq k D|s-t| \quad, \quad W_{p}\left(\rho_{0}, \rho_{t}\right) \leq k t W_{p}\left(\rho_{0}, \rho_{1}\right) .
$$

Proof. Denote by $d$ the geodesic distance in $\bar{\Omega}$, that is,

$$
\left.d(x, y):=\inf \left\{\int_{0}^{1}|\dot{\gamma}(s)| d s: \gamma \in \operatorname{Lip}([0,1], \bar{\Omega}), \gamma(0)=x, \gamma(1)=y\right\}\right\} .
$$

Since $\Omega$ is connected and $C^{0,1}$, there exists a constant $k \geq 1$ such that

$$
|x-y| \leq d(x, y) \leq k|x-y| .
$$

For every pair $(x, y) \in \bar{\Omega} \times \bar{\Omega}$, we select a geodesic curve $S_{x y}$ between $x$ and $y$ (not unique in general) and we denote by $[x, y]_{t}$ the unique point $z \in S_{x y}$ such that $d(x, z)=t d(x, y)$ (notice that $\left[x,\left.y\right|_{t}=(1-t) x+t y\right.$ if $\Omega$ is convex). This selection process can be done so that the map $(t, x, y) \in[0,1] \times \bar{\Omega} \times \bar{\Omega} \mapsto[x, y]_{t} \in \bar{\Omega}$ is Borel regular and satisfies, for every $0 \leq s<t \leq 1$, the properties:

$$
[x, y]_{t}=\left[[x, y]_{s}, y\right]_{t-s} \quad, \quad d\left([x, y]_{s},[x, y]_{t}\right)=|t-s| d(x, y) .
$$

In particular, from (2.15) and (2.16) , we deduce that, for every $(s, t, x, y) \in[0,1]^{2} \times$ $\bar{\Omega}^{2}$,

$$
\left|[x, y]_{s}-[x, y]_{t}\right| \leq d\left([x, y]_{s},[x, y]_{t}\right)=|s-t| d(x, y) \leq k|s-t||x-y| .
$$

Let $\gamma$ be an optimal transport plan for $W_{p}\left(\rho_{0}, \rho_{1}\right)$ and set for every $s, t \in[0,1]$ :

$$
\gamma_{s, t}=\Pi_{s, t}^{\sharp}(\gamma) \quad \text { where } \quad \Pi_{s, t}(x, y):=\left([x, y]_{s},[x, y]_{t}\right) .
$$

Then we define $\rho_{t}$ to be the second marginal of $\gamma_{0, t}$. Clearly such a definition agrees with $\rho_{0}$ and $\rho_{1}$ for $t=0$ and $t=1$ respectively, and moreover we have that, for every $s, t$, the marginals of $\gamma_{s, t}$ coincide with $\rho_{s}$ and $\rho_{t}$, that is, $\gamma_{s, t} \in \Gamma\left(\rho_{s}, \rho_{t}\right)$. Therefore, by (2.17), we have

$$
\begin{aligned}
W_{p}^{p}\left(\rho_{s}, \rho_{t}\right) & \leq \int_{\bar{\Omega} \times \bar{\Omega}}|x-y|^{p} \gamma_{s, t}(d x d y)=\int_{\bar{\Omega} \times \bar{\Omega}}\left|[x, y]_{s}-[x, y]_{t}\right|^{p} \gamma(d x d y) \\
& \leq k^{p}|s-t|^{p} \int_{\bar{\Omega} \times \bar{\Omega}}|x-y|^{p} \gamma(d x d y)=k^{p}|s-t|^{p} W_{p}^{p}\left(\rho_{0}, \rho_{1}\right),
\end{aligned}
$$

yielding in particular (2.14) if we take $s=0$. On the other hand, recalling (1.4) and (2.17), we obtain

$$
W_{\infty}\left(\rho_{s}, \rho_{t}\right) \leq \sup _{(x, y) \in \bar{\Omega} \times \bar{\Omega}}\left|[x, y]_{s}-[x, y]_{t}\right| \leq k D|t-s| .
$$

Proof of Proposition 1.1. Let $x \in \Omega \backslash N$ where $N$ is the $\mu$ negligible subset defined in Lemma 2.1. Setting $y=T_{p}(\mu, \nu)(x)\left(=T_{\nu}(x)\right)$, we deduce from (2.1), (2.2) and the lowerbound $f \geq a$ on $\Omega$ that

$$
\left|z-T_{\nu}(z)\right|^{p} f(z) \geq a \psi_{p}(x, y, z) \text { for } \mu \text {-a.e. } z \in \Omega \text {. }
$$


Thus, after integrating with respect to $z \in \Omega$ and by the definitions (2.10) and (2.11), we obtain for all $x \in \Omega \backslash N$ :

$$
\begin{aligned}
W_{p}^{p}(\mu, \nu) & \geq a\left|x-T_{\nu}(x)\right|^{p+d} \omega\left(\left|x-T_{\nu}(x)\right|\right) \\
& \geq a\left|x-T_{\nu}(x)\right|^{p+d} \inf \{\omega(t): 0<t \leq D\} .
\end{aligned}
$$

Thus, taking the essential supremum in $x$, we are led to

$$
\frac{W_{p}^{p}(\mu, \nu)}{\left\|\operatorname{Id}-T_{\nu}\right\|_{\infty, \Omega}^{p+d}} \geq a \inf \{\omega(t), 0<t \leq D\}
$$

that is (1.3) if we set

$$
\left(c_{p, d}(\Omega)\right)^{-1}=\inf \{\omega(t): 0<t \leq D\} .
$$

So to complete the proof of the proposition, it is enough to show that the right hand side in (2.19) is strictly positive. Since the function $\omega(t)$ is lower semicontinuous on $(0, D]$ and, by Lemma 2.4. satisfies $\liminf _{t \rightarrow 0^{+}} \omega(t)=\omega_{p, d}(\Omega)>0$, we are reduced to checking that $\omega(t)>0$ for $0<t \leq D$. For such a $t$, by the lower semicontinuity of $\rho$, we have $\omega(t)=\rho(x, y)$ for suitable $x, y \in \bar{\Omega}$ with $|x-y|=t$. By the convexity assumption, $\Omega$ intersects the convex open set $x+Q_{p}\left(\frac{y-x}{|y-x|}\right)$ where the function $\psi_{p}(x, y, \cdot)$ is strictly positive. As this set has a positive Lebesgue measure, it follows that $\omega(t)=\rho(x, y)>0$.

Proof of Theorem 1.2, Let us define for every $\delta>0(\delta$ not larger than the diameter $D$ of $\Omega$ ):

$$
\begin{aligned}
& \alpha(\delta):=\inf \left\{\frac{1}{a} W_{p}^{p}(\mu, \nu):(\mu, \nu) \in \mathcal{P}_{\mathrm{ac}}(\Omega) \times \mathcal{P}(\bar{\Omega}), \frac{d \mu}{d x} \geq a, W_{\infty}(\mu, \nu)=\delta\right\}, \\
& \beta(\delta):=\inf \left\{\frac{1}{a} W_{p}^{p}(\mu, \nu):(\mu, \nu) \in \mathcal{P}_{\mathrm{ac}}(\Omega) \times \mathcal{P}(\bar{\Omega}), \frac{d \mu}{d x} \geq a, W_{\infty}(\mu, \nu) \geq \delta\right\} .
\end{aligned}
$$

Proving (1.5) is equivalent to showing the following inequality:

$$
\inf \left\{\frac{\alpha(\delta)}{\delta^{p+d}}: 0<\delta \leq D\right\}\left(:=\left(C_{p, d}(\Omega)\right)^{-1}\right)>0 .
$$

We observe that, by the definition (1.4), (2.18) implies that

$$
W_{p}^{p}(\mu, \nu) \geq a W_{\infty}^{p+d}(\mu, \nu) \inf \{\omega(t), 0<t \leq D\} .
$$

Therefore, by the definition of $\alpha(\delta)$ and Lemma 2.4 we infer that

$$
\liminf _{\delta \rightarrow 0} \frac{\alpha(\delta)}{\delta^{p+d}} \geq \lim _{\delta \rightarrow 0}(\inf \{\omega(t), 0<t \leq \delta\})=\omega_{p, d}(\Omega)>0 .
$$

Fixing $t_{0} \in(0,1)$, we deduce that there exists a suitable $\delta_{0}>0$ such that

$$
\frac{\alpha(\delta)}{\delta^{p+d}} \geq t_{0} \omega_{p, d}(\Omega) \quad \text { for all } \delta \leq \delta_{0} .
$$

Now we claim that there exists a constant $C \geq 1$ such that:

$$
\beta(\delta) \leq \alpha(\delta) \leq C \beta(\delta) \quad \text { for every } \delta \leq D .
$$

As $\beta$ is nondecreasing, we infer from (2.22) that $C \alpha(\delta) \geq \alpha\left(\delta_{0}\right)$ for $\delta \geq \delta_{0}$. This together with (2.21) allows us to conclude (2.20) since we have

$$
\inf \left\{\frac{\alpha(\delta)}{\delta^{p+d}}: 0<\delta \leq D\right\} \geq \min \left\{t_{0} \omega_{p, d}(\Omega), \frac{\alpha\left(\delta_{0}\right)}{C D^{p+d}}\right\} .
$$


In order to finish the proof of Theorem 1.2, let us now show that the claim (2.22) holds true with $C=k^{p}$ where $k$ is the constant appearing in Lemma 2.5. Assume that $k^{p} \beta(\delta)<\alpha(\delta)$ for some $\delta \in[0, D]$. Then, by the definition of $\beta(\delta)$, there exists an admissible pair $(\mu, \nu)$ where $\mu=f d x, a>0$ with $f \geq a$ a.e. such that:

$$
\beta(\delta) \leq \frac{W_{p}^{p}(\mu, \nu)}{a}<\frac{\alpha(\delta)}{k^{p}} \quad \text { and } \quad W_{\infty}(\mu, \nu) \geq \delta .
$$

Owing to Lemma 2.5. we consider a Lipschitz curve $t \in[0,1] \mapsto \rho_{t} \in \mathcal{P}(\bar{\Omega})$, where $\mathcal{P}(\bar{\Omega})$ is equipped with the $W_{\infty}$ distance, such that $\rho_{0}=\mu$ and $\rho_{1}=\nu$. Then the function $h(t):=W_{\infty}\left(\mu, \rho_{t}\right)$ is continuous on [0,1], vanishes at 0 and by (2.23) satisfies $h(1) \geq \delta$. Thus there exists a suitable $\bar{t} \in(0,1]$ such that $h(\bar{t})=\delta$ and recalling the definition of $\alpha(\delta)$, we find that $W_{p}^{p}\left(\mu, \rho_{\bar{t}}\right) \geq a \alpha(\delta)$ whereas, by (2.14) and (2.23), $W_{p}^{p}\left(\mu, \rho_{\bar{t}}\right) \leq k^{p} W_{p}^{p}(\mu, \nu)<a \alpha(\delta)$. This contradicts the definition of $\alpha(\delta)$ and thus (2.22) is proved.

Remark 2.6. The constants $c_{p, d}(\Omega)$ and $C_{p, d}(\Omega)$ found in the proofs above turn out to be directly correlated with the inverse of the constant $\omega_{p, d}(\Omega)$ introduced in Lemma 2.4. This positive real coincides with the minimum over $u \in S^{d-1}$ of the function

$$
I_{p}(u):=\max \left\{\int_{Q_{p}\left(e_{1}\right)} \overline{\psi_{p}}(u, w) d w, \int_{Q_{p}\left(e_{1}\right)} \overline{\psi_{p}}(u, u+w) d w\right\},
$$

$e_{1}$ being the first element of the canonical basis. Unfortunately $I_{p}(u)$ converges to zero as $p \rightarrow 1_{+}$, making our constants $c_{p, d}(\Omega)$ and $C_{p, d}(\Omega)$ blow-up.

As we already claimed in remark 1.5. (1.3) does not remain true for $p=1$ in general. This fact is corroborated by the following example.

Example 2.7. Take $p=d=1, \Omega=] 0,1[$ and consider the following measures:

$$
\mu:=\mathcal{L}_{\mid[0,1]}, \quad \nu_{\varepsilon}=(1+\varepsilon) \mathcal{L}_{\left.\left.\right|_{\left[0, \frac{1}{1+\varepsilon}\right.}\right]} .
$$

Then, for every $\varepsilon>0$, the map $T_{\varepsilon}^{o}: x \mapsto \frac{x}{1+\varepsilon}$ is the unique nondecreasing map pushing forward $\mu$ on $\nu_{\varepsilon}$ and it is optimal, i.e.:

$$
W_{1}\left(\mu, \nu_{\varepsilon}\right)=\int_{0}^{1}\left|x-T_{\varepsilon}^{0} x\right| d x=\frac{1}{2}\left(\frac{\varepsilon}{1+\varepsilon}\right) .
$$

It is easy to check that another optimal transport map relative to $W_{1}\left(\mu, \nu_{\varepsilon}\right)$ is given by:

$$
T_{\varepsilon}(x)=x \quad \text { if } \quad x \in\left[0, \frac{1}{1+\varepsilon}\right] \quad, \quad T_{\varepsilon}(x)=\frac{1}{\varepsilon} x-\frac{1}{\varepsilon(1+\varepsilon)} \quad \text { if } x \in\left[\frac{1}{1+\varepsilon}, 1\right] .
$$

The maximal transport distance is $\frac{1}{1+\varepsilon}$, and so

$$
W_{1}\left(\mu, \nu_{\varepsilon}\right)=\frac{\varepsilon(1+\varepsilon)}{2}\left\|\operatorname{Id}-T_{\varepsilon}\right\|_{L^{\infty}(\Omega)}^{2},
$$

which, as $\varepsilon \rightarrow 0$, confirms that the estimate in Proposition 1.1 does not hold true for $p=1$. However, no contradiction occcurs if we substitute $T_{\varepsilon}$ with the monotone transport $T_{\varepsilon}^{0}$ since we have

$$
W_{1}\left(\mu, \nu_{\varepsilon}\right)=\frac{1+\varepsilon}{2 \varepsilon}\left\|\mathrm{Id}-T_{\varepsilon}^{0}\right\|_{L^{\infty}(\Omega)}^{2} .
$$


In fact, in the one dimensional case, the estimate (1.3) holds true for $p=1$ if we choose $T_{\nu}$ to be the unique nondecreasing transport map.

Proposition 2.8. Let $d=1$ and let $\Omega$ be a bounded interval of $\mathbb{R}$ on which $f \geq a(a>0)$. Then, for every $\nu$ supported in $\bar{\Omega}$, the unique monotone map $T_{\nu}$ transporting $f d x$ on $\nu$ satisfies for every $p \geq 1$

$$
\left\|I d-T_{\nu}\right\|_{L^{\infty}(\Omega)}^{p+1} \leq \frac{p+1}{a} W_{p}^{p}(\mu, \nu) .
$$

A consequence of Proposition 2.8 is that, if $\Omega$ is a bounded interval of $\mathbb{R}$, then the inequality (1.5) of Theorem 1.2 holds true for $p \geq 1$ with $C_{p, 1}(\Omega)=p+1$.

Proof. The monotonocity of the map $T_{\nu}$ implies that it is optimal with respect to all distances $W_{p}$ with $p \geq 1$ (see for instance [3]). In particular, we obtain that if $x \in \Omega$ and $y=T_{\nu} x$, then a.e. $z$ in the interval $(x, y)$ (resp. $\left.(y, x)\right)$ satisfies the inequality $\left|z-T_{\nu} z\right|^{p} \geq|z-y|^{p}$. Thus, after integrating in $z$, we obtain that for a.e. $x \in \Omega$ :

$$
W_{p}^{p}(f, \nu) \geq \frac{a}{p+1}\left|x-T_{\nu} x\right|^{p+1} .
$$

We deduce (2.24) by taking the essential supremum in the right hand side.

\section{REFERENCES}

[1] L. A. Caffarelli, Interior $W^{2, p}$ estimates for the solution of Monge-Ampère equation, Ann. of Math. (2) 131, 1 (1990), 135-150. MR1038360 (91f:35059)

[2] L. A. Caffarelli, Some regularity properties of solutions of Monge-Ampère equation, Comm. Pure Appl. Math. 44, 8-9 (1991), 965-969. MR1127042 (92h:35088)

[3] L. Ambrosio, Lecture notes on optimal transport problems. Mathematical aspects of evolving interfaces (Funchal, 2000), 1-52, Lecture Notes in Math., 1812, Springer, Berlin (2003). MR2011032

[4] L. Ambrosio, A. Pratelli, Existence and stability results in the $L^{1}$ theory of optimal transportation. Optimal transportation and applications (Martina Franca, 2001), 123-160, Lecture Notes in Math., 1813, Springer, Berlin (2003). MR2006307

[5] G. Bouchitté, C. Jimenez, M. Rajesh, Asymptotique d'un problème de positionnement optimal, C. R. Acad. Sci. Paris, Ser. I 335 (2002), 835-858. MR.1947712 (2003k:49029)

[6] L. Caffarelli, M. Feldman, R. J. McCann, Constructing optimal maps for Monge's transport problem as a limit of strictly convex costs, J. Amer.Math. Soc., 15, (2002). MR 1862796 (2003b:49042)

[7] L. C. Evans, Partial differential equations and Monge-Kantorovich mass transfer. Current developments in mathematics (1997) (Cambridge, MA), 65-126. MR1698853(2000e:49001)

[8] L. C. Evans, W.Gangbo, Differential Equation Methods for the Monge-Kantorovich Mass Transfer Problem, Memoirs AMS, 658 (1999). MR 1464149(99g:35132)

[9] W. Gangbo and R. J. McCann, The geometry of optimal transportation Acta Math. 177, 2 (1996), 113-161. MR.1440931 (98e:49102)

[10] L. V. Kantorovich, On the transfer of masses, Dokl. Akad. Nauk. SSSR 37 227-229 (1942).

[11] G. Monge, Mémoire sur la theorie des deblais et des remblais, Histoire de l'Académie Royale des Sciences, Paris (1781).

[12] V.N. Sudakov, Geometric problems in the theory of infinite dimensional distributions, Proc. Steklov Inst. Math., 141 (1979), 1-178. MR0530375 (80e:60052)

[13] C. Villani, Topics in optimal transportation, Graduate Studies in Mathematics, 58, AMS (2003). MR 1964483 (2004e:90003) 
UfR Sciences, Université du Sud-Toulon-Var, BP20132, 83957 La Garde Cedex, FRANCE

E-mail address: bouchitte@univ-tln.fr

UfR Sciences, Université du Sud-Toulon-Var, BP20132, 83957 La Garde Cedex, FRANCE

E-mail address: c.jimenez@sns.it

Departemento de Matematica, Facultad de Ciencias Fisicas y Matematicas, UniverSidad de Concepcion, Casilla 160-C. Concepcion, Chile

E-mail address: rmahadevan@udec.cl 